\title{
A COMMENT ON THE PAPER 'ON THE INTENSITY OF CROSSINGS BY A SHOT NOISE PROCESS'
}

\author{
PANAGIOTIS KONSTANTOPOULOS, ${ }^{*}$ University of California, Berkeley
}

In the paper [3] Hsing derives a formula for the intensity of upcrossings of a certain level $u$ by the output process $\{X(t), t \in \mathbb{R}\}$ from a linear filter with deterministic impulse response $\{h(t), t \in \mathbb{R}\}$ and Poisson shot noise as input. More specifically, it is proven that if

$$
X(t)=\int_{(-\infty, t]} h(t-s) N(d s)
$$

where $N$ is a Poisson process with rate $\lambda$ and $h$ is a non-increasing function from $[0, \infty)$ into $[0, \infty)$, then the rate of upcrossings of a certain level $u>0$ by $X$ is

$$
\lambda P\{u-h(0)<X(0-) \leqq u\}
$$

The proof uses explicitly some independence properties of $N$.

However, formula (2) has nothing to do with independence or any other characteristic properties of the Poisson process but is solely due to stationarity. It is therefore valid in much more generality for any stationary input, provided that $P$ in (2) is replaced by its Palm transformation $P^{0}$ with respect to the input point process $N$. (For the notion of the Palm distribution see [1], [2], [4].) In other words we make the following claim.

Proposition. Let $\left(T_{n}, h_{n}\right), n \in \mathbb{Z}$ be a stationary marked point process defined on some probability space $(\Omega, F, P)$. Let $N$ denote the underlying point process (with points $\cdots<$ $\left.T_{0} \leqq 0<T_{1}<T_{2}<\cdots\right)$ and let $\lambda$ be its rate $(0<\lambda<\infty)$. The marks $h_{n}$ take values in the space of non-increasing non-negative functions on $[0, \infty)$. Define

$$
X(t)=\sum_{n: t \leq T_{n}} h_{n}\left(t-T_{n}\right), \quad t \in \mathbb{R} .
$$

Then the intensity of upcrossings of $u$ by $X$ is

$$
\lambda P^{0}\left\{u-h_{0}(0)<X(0-) \leqq u\right\},
$$

where $P^{0}$ is the Palm transformation of $P$ with respect to $N$.

Proof. $X$ crosses $u$ at time $t$ if $X(t-) \leqq u$ and $X(t+)>u$. This can happen only if $t=T_{n}$ since these are the only increase points of $X$. Let $A_{n}=\left\{X\left(T_{n}-\right) \leqq u, X\left(T_{n}+\right)>u\right\}=$ $\left\{X\left(T_{n}-\right) \leqq u, X\left(T_{n}-\right)+h_{n}(0)>u\right\}=\left\{u-h_{n}(0)<X\left(T_{n}-\right) \leqq u\right\}$. The intensity of upcrossings of $u$ is (by definition):

$$
E \sum_{n \in \mathbb{Z}} 1_{A_{n}} 1\left(T_{n} \in[0,1]\right)
$$

where $E$ denotes expectation with respect to $P$. Observing that the sequence of events $A_{n}$ is stationary under the measure $P^{0}$ and letting $E^{0}$ denote expectation with respect to $P^{0}$, we can

Received 3 August 1988; revision received 6 December 1988.

* Postal address: Department of Electrical Engineering and Computer Sciences and Electronics Research Laboratory, University of California, Berkeley, CA 94720, USA. 
apply Campbell's formula to (5) (see [1], [2]) to see that (5) equals:

$$
\lambda E^{0} \int_{\mathbb{R}} 1_{A_{0}} 1(t \in[0,1]) d t=\lambda P^{0}\left(A_{0}\right)=\lambda P^{0}\left\{u-h_{0}(0)<X(0-) \leqq u\right\}
$$

(since $P^{0}\left\{T_{0}=0\right\}=1$ ). This proves the claim.

We can now see that if we take $N$ to be a Poisson process and $h_{n}=h$ for all $n$, where $h$ is a deterministic function, then process (3) is equal to (1) and formula (4) yields (2) since $P^{0}$, for a Poisson process, is equal to $P$.

\section{References}

[1] Baccelli, F. and Brémaud, P. (1987) Palm Probabilities and Stationary Queues. Lecture Notes in Statistics 41, Springer-Verlag, Berlin.

[2] Franken, P., König, D., Arndt, U. and Schmidt, V. (1982) Queues and Point Processes. Akademie-Verlag, Berlin.

[3] Hsing, T. (1987) On the intensity of crossings by a shot noise process. Adv. Appl. Prob. 19, 743-745.

[4] Kallenberg, O. (1983) Random Measures. Akademie-Verlag, Berlin. 